\title{
A retrospective TBNET assessment of linezolid safety, tolerability and efficacy in multidrug-resistant tuberculosis
}

\author{
G.B. Migliori, B. Eker, M.D. Richardson, G. Sotgiu, J-P. Zellweger, A. Skrahina, \\ J. Ortmann, E. Girardi, H. Hoffmann, G. Besozzi, N. Bevilacqua, D. Kirsten, \\ R. Centis and C. Lange for the TBNET Study Group
}

ABSTRACT: Linezolid is used to treat patients with multidrug-resistant (MDR)/extensively drugresistant (XDR)-tuberculosis (TB) cases, although clinical data on its safety, tolerability and efficacy are lacking.

We performed a retrospective, nonrandomised, unblinded observational study evaluating the safety and tolerability of linezolid at $600 \mathrm{mg}$ q.d. or b.i.d. in MDR/XDR-TB treatment in four European countries. Efficacy evaluation compared end-points of 45 linezolid-treated against 110 linezolid-nontreated cases.

Out of $195 \mathrm{MDR} / \mathrm{XDR}$-TB patients, 85 were treated with linezolid for a mean of 221 days. Of these, $35(41.2 \%)$ out of 85 experienced major side-effects attributed to linezolid (anaemia, thrombocytopenia and/or polyneuropathy), requiring discontinuation in 27 (77\%) cases. Most side-effects occurred after 60 days of treatment. Twice-daily administration produced more major side-effects than once-daily dosing $(p=0.0004)$, with no difference in efficacy found. Outcomes were similar in patients treated with/without linezolid $(p=0.8)$, although linezolid-treated cases had more first-line $(p=0.002)$ and second-line $(p=0.02)$ drug resistance and a higher number of previous treatment regimens $(4.5$ versus $2.3 ; p=0.07$ ).

Linezolid $600 \mathrm{mg}$ q.d. added to an individualised multidrug regimen may improve the chance of bacteriological conversion, providing a better chance of treatment success in only the most complicated MDR/XDR-TB cases. Its safety profile does not warrant use in cases for which there are other, safer, alternatives.

KEYWORDS: Efficacy, extensively drug-resistant tuberculosis, linezolid, multidrug-resistant tuberculosis, safety, tolerability

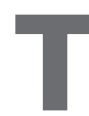

uberculosis (TB) is a leading cause of death worldwide. The World Health Organization (WHO) estimates that approximately 9.2 million new TB cases and 1.5 million TB-related deaths occurred in 2006 [1]. The prevalence of multidrug-resistant (MDR) Mycobacterium tuberculosis (defined as in vitro resistance to isoniazid and rifampicin, the two most potent first-line drugs for TB treatment) is increasing globally $[2,3]$. MDR strains are now found in more than $15 \%$ of all TB cases in some countries of the former Soviet Union, Israel and areas of China [4-6]. M. tuberculosis resistant to second-line drugs is also emerging. Cases of extensively drug-resistant (XDR) TB (defined by the $\mathrm{WHO}$ as in vitro resistance to isoniazid and rifampicin plus any fluoroquinolone and at least one of the injectable drugs: capreomycin, kanamycin or amikacin) have been described in most regions of the world $[7,8]$. This article is part of a larger effort by the Tuberculosis Network European Trials Group (TBNET) to characterise the clinical epidemiology of MDRand XDR-TB in Europe and thus strengthen the evidence base for clinical and public health recommendations for MDR- and XDR-TB control.

Treatment outcomes for complicated MDR-TB cases (those with additional resistance beyond isoniazid and rifampicin) and cases with XDR-TB are poor [9-15]. Thus, information on safety, tolerability and efficacy of other antibiotics potentially useful in treatment is urgent to improve individual outcomes and control the spread of MDR- and XDR-TB. In vitro and pharmacological data suggest that linezolid, an oxazolidinone antibiotic, could be useful in
AFFILIATIONS

For author affiliations and additional participating members of the Tuberculosis Network European Trials Group (TBNET) Study Group, please see the Acknowledgements section.

CORRESPONDENCE

C. Lange

Tuberculosis Network European Trials Group (TBNET), Clinical Infectious Diseases

Research Center Borstel

Parkallee 35

23845 Borstel

Germany

E-mail: clange@zz-borstel.de

Received:

Jan 202009

Accepted after revision:

Feb 242009

First published online:

March 122009 
treating mycobacterial infections, including MDR-TB [16-23]. However, clinical experience with linezolid for purposes outside the drug's approved uses has been restricted mainly to case reports in nontuberculous mycobacterial diseases [2429]. Linezolid is used in both Eastern and Western Europe to treat MDR/XDR-TB, a purpose outside the drug's approved uses, but treatment costs are high. In a recent epidemiological study on MDR-TB in Germany [15], linezolid was widely used: $33.8 \%$ of the patients reported side-effects, with $56 \%$ of those experiencing severe anaemia. However, these data and other previous studies [22, 30-33] did not provide sufficient detail to evaluate safety, tolerability and efficacy of linezolid in MDR/ XDR-TB treatment. The aim of this study was to evaluate the safety, tolerability and efficacy of linezolid using a sizable cohort of patients with MDR/XDR-TB from four European countries.

\section{METHODS}

\section{Study design}

Safety and tolerability were analysed in this retrospective cohort study of MDR/XDR-TB patients from 21 participating centres in Belarus, Germany, Italy and Switzerland from 2001 to 2007. To assess the efficacy of linezolid in MDR/XDR-TB treatment, we compared only patients who had definitive treatment end-points recorded (cured, completed, died or failed). Patients who were still on treatment at the time of the data collection were excluded from the analysis as no followup information was available for these cases.

The data collection tool was pre-tested in Germany [15], providing the following information: previous treatment, HIV status, drug-resistance profile, time to sputum and culture conversion, length of hospital stay, adverse effects (yes/no), treatment interruption (yes/no) and treatment outcome. This tool was refined to include linezolid dosage and details on adverse effects, linezolid reintroduction (including dosage) and anti-TB drugs used in treatment regimens. The final dataset included complete information on 59 of the $74 \mathrm{MDR} /$ XDR-TB cases from Germany and on 26 additional cases from Italy, Belarus and Switzerland.

Approval for collection of study data was provided by the ethical committee of the clinical coordinating centre at Sondalo Hospital in Sondalo, Italy, and the statistical coordinating centre, Fondazione S. Maugeri in Tradate, Italy. All participating centres complied with national regulations and organisational requirements for human subject protection. All data were coded and individual identifiers were available only to treating physicians.

\section{Definitions}

Standard treatment outcome definitions as proposed by LASERSON et al. [34] were used as previously described [11, 35].

A patient who completed treatment and was consistently culture negative with at least five results for the final 12 months of treatment was defined as cured. If bacteriological results were lacking (i.e. fewer than five cultures performed), the case was defined as treatment completed. Treatment failure was defined as two or more positive cultures in the last 12 months of treatment, or if a medical decision was made to terminate treatment due to poor response or adverse events.
Safety and tolerability end-points included major and minor side-effects. A major side-effect was defined as any adverse reaction that resulted in temporary or permanent discontinuation of linezolid, whereas a minor side-effect required only dose adjustment and/or addition of concomitant treatment. All patients in the cohort treated with linezolid were included in the safety and tolerability analysis.

Efficacy end-points included time to and proportion of sputum smear and culture conversions, and treatment outcome. Sputum conversion was defined as two consecutive negative sputum smears in patients who were smear positive at diagnosis. Time to culture conversion was defined as time from treatment start to date of the first of two consecutive negative cultures.

\section{Data collection}

Data were extracted from clinical records of all cultureconfirmed MDR/XDR-TB cases diagnosed consecutively by the participating centres and verified by the study review panel. Drug susceptibility testing (DST) for first- and secondline anti-TB drugs was performed by laboratories qualityassured by the WHO's Supranational Reference Laboratories (Rome/Milan, Borstel, Stockholm) [9-11]. All participating centres performed sputum smears weekly until negative, and then monthly. Cultures were performed monthly.

\section{Treatment regimens}

In all countries, regimens to treat MDR/XDR-TB cases were tailored to DST results according to WHO recommendations, using fluoroquinolones, injectable agents and other second-line oral agents. Linezolid was available without any limitation, except in Belarus where it was prescribed for a maximum of 3 months to optimise the use of the drug, given its limited availability in the country.

\section{Statistical analyses}

Categorical variables were compared by Chi-squared test and continuous variables by t-test for unpaired data. A p-value of $\leqslant 0.05$ was considered statistically significant. Data were collected on standardised e-forms and analysed using Stata 9.0 (StataCorp, Stata Statistical Software Release 9, College Station, TX, USA).

\section{RESULTS}

Within the cohort of 195 MDR/XDR-TB cases, 85 patients were treated with an individualised regimen including linezolid and 110 were treated without linezolid. In the linezolid group, 45 patients had a definitive treatment end-point and 40 patients did not: one $(2.5 \%)$ out of 40 defaulted, one $(2.5 \%)$ transferred out and $38(95 \%)$ were still on treatment.

\section{Safety and tolerability}

Safety and tolerability were evaluated for all 75 MDR-TB patients $(88.2 \%)$ and 10 XDR-TB patients $(11.8 \%)$ treated with linezolid. Of these, 28 patients received linezolid at $600 \mathrm{mg}$ q.d. and 57 patients received $600 \mathrm{mg}$ b.i.d. (1,200 mg). No differences in the main sociodemographic variables or in drugresistance profiles were observed between these two groups (data not shown). 
Linezolid was administered for a mean \pm SD period of $222 \pm 249$ days (median 93 days).

Adverse events appeared after a median of 69 days (range 1-596 days) of linezolid treatment. In total, 35 (41.2\%) out of 85 patients experienced 52 episodes of side-effects attributable to linezolid. Of the 35 patients experiencing side-effects, 27 $(77.1 \%)$ experienced major side-effects requiring temporary or permanent discontinuation of linezolid, whereas eight $(22.9 \%)$ patients experienced minor side-effects.

Side-effects were primarily represented by anaemia (23 (44.2\%) out of 52 episodes). Out of 23 patients experiencing anaemia, five $(21.7 \%)$ were severely affected, requiring blood transfusion as the haemoglobin level was $<8 \mathrm{mg} \cdot \mathrm{dL}^{-1}$; two of these patients had haemolytic anaemia. Other side-effects were thrombocytopenia (seven (13.5\%) out of 52), nausea/vomiting (four $(7.7 \%)$ out of 52 ) and polyneuropathy (three (5.7\%) out of 52 ). In eight $(29.6 \%)$ out of 27 patients experiencing major sideeffects, linezolid was successfully reintroduced and in 19 $(70.4 \%)$ out of 27 it was permanently discontinued. No linezolid-related deaths were observed.

A significant difference in side-effects was observed between those receiving $600 \mathrm{mg}$ versus $1,200 \mathrm{mg}$ daily. Only four $(14.3 \%)$ out of 28 patients receiving $600 \mathrm{mg}$ of linezolid experienced any side-effects versus 31 (54.4\%) out of the 57 who received $1,200 \mathrm{mg}$ ( $\mathrm{p}=0.0004$; fig. 1 ). Patients prescribed linezolid at $600 \mathrm{mg}$ daily had a lower risk of major side-effects as well as a lower risk of any side-effects than those prescribed 1,200 mg (table 1).

\section{Efficacy end-points analysis}

No significant differences were found in efficacy between 45 patients treated with linezolid and 110 patients not treated with linezolid for the main demographic and clinical variables. There were 41 MDR-TB cases (91.1\%) among the 45 linezolidtreated patients and 102 among 110 patients not treated with linezolid $(92.7 \% ; \mathrm{p}=0.83)$, whereas there were four $(8.9 \%)$ and

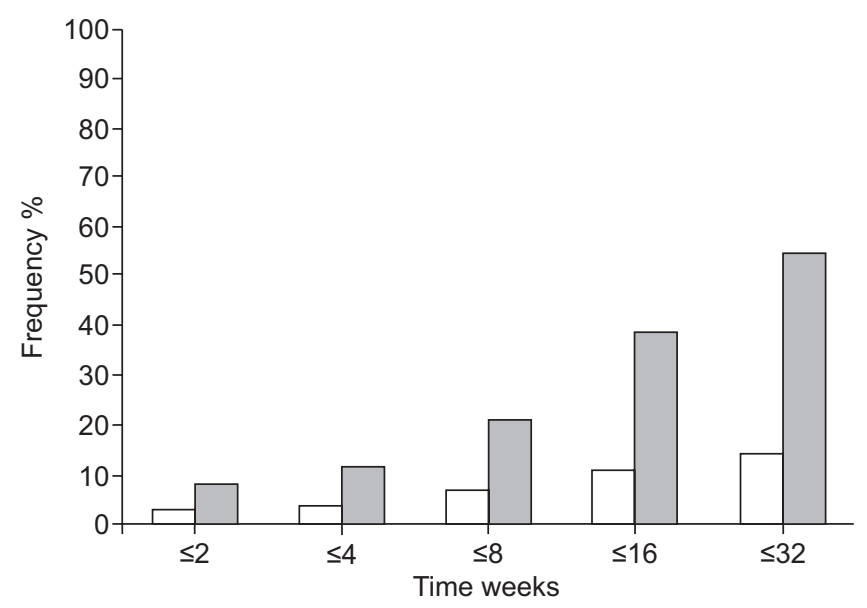

FIGURE 1. Frequency of adverse effects attributed to linezolid during combined treatment against multidrug-resistant/extensively drug-resistant tuberculosis at different time-points after treatment initiation with a $600 \mathrm{mg}$ q.d. or a $600 \mathrm{mg}$ b.i.d. regimen (denominator is the total number of individuals per group). $\square: 600$ mg q.d., $\mathrm{n}=28$; $\square: 600$ mg b.i.d., $\mathrm{n}=57$. eight $(7.3 \% ; p=0.82)$ XDR-TB patients in the two groups, respectively. The mean number of first-line drugs to which those treated with or without linezolid were resistant was 4.3 and 3.8, respectively $(p=0.002)$, with 1.5 and 0.9 for second-line drugs, respectively $(p=0.02)$. Both groups included retreatment patients, but the mean number of treatment regimens previously prescribed ( $>1$ month) was almost doubled in the linezolid-treated group (4.5 versus 2.3 , respectively; $\mathrm{p}=0.07$ ). Both linezolid-treated and nontreated patients were prescribed a mean number of five drugs.

Those treated with linezolid took significantly longer for both sputum smear and culture conversion than those without, but they achieved a higher (although nonsignificant) percentage of conversion overall (table 2). The mean $\pm S D$ time to smear conversion was $102.9 \pm 74$ days in the linezolid group and $65.4 \pm 80.1$ days in the non-linezolid group $(p=0.007)$, whereas the mean $\pm \mathrm{SD}$ time to culture conversion was $109 \pm 71$ and $69 \pm 63$ days, respectively $(\mathrm{p}=0.0007)$. The proportion achieving sputum smear conversion was $69 \%$ for the linezolid group versus $54 \%$ in the non-linezolid group (relative risk (RR) 1.28; $95 \%$ confidence interval $(\mathrm{CI}) 0.99-1.6 ; \mathrm{p}=0.08)$. The proportion achieving culture conversion was 87 versus $78 \%$ (RR $1.1 ; 95 \%$ CI $0.95-1.28 ; p=0.22)$. There was no significant difference in treatment outcomes for those with definitive treatment endpoints: $36(80 \%)$ out of 45 linezolid-treated patients achieved treatment success versus $90(81.8 \%)$ out of 110 non-linezolidtreated patients $(p=0.88)$, and there was no difference in the risk of failure or death between the two groups.

Efficacy was similar for patients who were prescribed $600 \mathrm{mg}$ versus 1,200 mg of linezolid: the proportion achieving sputum smear conversion was $71.4 \%$ in the $600 \mathrm{mg}$ versus $67.7 \%$ in the $1,200 \mathrm{mg}$ group $(\mathrm{p}=0.8)$. The proportion achieving culture conversion was $92.9 \%$ in those receiving $600 \mathrm{mg} \cdot$ day $^{-1}$ versus $83.9 \%$ in the $1,200 \mathrm{mg} \cdot$ day $^{-1}$ group $(\mathrm{p}=0.4)$. The mean $\pm \mathrm{SD}$ time to sputum smear conversion was $101.5 \pm 79.3$ days in the $600 \mathrm{mg} \cdot$ day $^{-1}$ group and $103.6 \pm 73.4$ days in the $1,200 \mathrm{mg} \cdot \mathrm{day}^{-1}$ group $(\mathrm{p}=0.9)$, whereas the mean $\pm \mathrm{SD}$ time to culture conversion was $109 \pm 74.6$ and $108.8 \pm 70$ days, respectively $(p=0.9)$. No significant difference in efficacy end-points (sputum and culture conversion, or positive treatment outcome) was observed related to the presence or absence of cavities on chest radiographs.

Efficacy end-point analysis stratified by resistance patterns When stratified by the number of drugs to which isolates were resistant, the proportion of sputum smear and culture converters in the linezolid group was higher than for the non-linezolid group for all strata, reaching statistical significance only for cases harbouring strains resistant to more than seven drugs ( $p=0.009$ for smear; $p=0.004$ for culture; table 3 ). There was no clear trend in time to conversion related to the number of drugs to which isolates were resistant (data not shown).

\section{Risk of adverse outcome}

The logistic regression analysis presented in table 4 showed a significant risk of adverse outcome (failure or death) related to increasing age, resistance to more than five drugs and the presence of XDR-TB, and was not influenced by linezolid treatment (crude odds ratios). Adjusted odds ratios, however, 


\begin{tabular}{|c|c|c|c|c|c|}
\hline \multirow[t]{2}{*}{ TABLE 1} & \multicolumn{5}{|c|}{$\begin{array}{l}\text { Safety and tolerability of linezolid in patients } \\
\text { treated for multidrug-resistant/extensively drug- } \\
\text { resistant tuberculosis in Belarus, Germany, Italy } \\
\text { and Switzerland, 2001-2007 }\end{array}$} \\
\hline & & Total & $600 \mathrm{mg}$ q.d. & 600 mg b.i.d. & p-value ${ }^{\#}$ \\
\hline \multicolumn{6}{|l|}{ Patients } \\
\hline Total $n$ & & 85 & 28 & 57 & \\
\hline No adverse ev & vent & $50(58.8)$ & $24(85.7)$ & $26(45.6)$ & 0.0004 \\
\hline Any adverse e & event & 35 (41.2) & $4(14.3)$ & $31(54.4)$ & 0.0004 \\
\hline Minor & & $8(9.4)$ & 0 & $8(14)$ & \\
\hline Major & & 27 (31.8) & $4(14.3)$ & $23(40.4)$ & 0.01 \\
\hline \multicolumn{6}{|l|}{ Episodes } \\
\hline Total $n$ & & 52 & 5 & 47 & \\
\hline Anaemia & & $23(44.2)$ & $3(60)$ & $20(42.5)$ & 0.44 \\
\hline Thrombocytop & penia & 7 (13.5) & $0(0)$ & $7(14.9)$ & \\
\hline Nausea/vomitir & & $4(7.7)$ & $1(20)$ & $3(6.4)$ & 0.25 \\
\hline Polyneuropath & & $3(5.8)$ & $1(20)$ & $2(4.3)$ & 0.13 \\
\hline Others & & $15(28.8)$ & $0(0)$ & $15(31.9)$ & \\
\hline
\end{tabular}

Data are presented as $n(\%)$, unless otherwise stated. ${ }^{\#}$ : comparison between $600 \mathrm{mg}$ q.d. group and $600 \mathrm{mg}$ b.i.d. group.

showed a significant risk of adverse treatment outcome only for increasing age and the presence of XDR-TB.

\section{DISCUSSION}

In the absence of new drugs for TB treatment, curing patients with MDR/XDR-TB critically depends on the pattern of drug resistance and on the availability of second- and third-line antiTB drugs that have reasonable safety, tolerability and efficacy $[13,14]$. Anecdotal reports suggest that linezolid may be an effective option to treat complicated cases of MDR/XDR-TB. However, serious concerns have been raised regarding safety and tolerability. Results of the five studies available with more than one case described suggest that adverse events may range from $43 \%$ [30] up to $80 \%$ or more [22, 29, 32, 33, 35]. Results of the present study augment findings of the previous study [15], where adverse effects were observed in $33.8 \%$ of the cases but no information on doses prescribed or details on side-effects were available.

Our results confirm a high frequency of major adverse sideeffects associated with linezolid, particularly in the higher $1,200 \mathrm{mg}$ dose and in treatment longer than 8 weeks. Although results from our study are more encouraging than most of those described previously [22, 30-33] and adverse events seemed reversible shortly after timely drug discontinuation or dose reduction [30], they are still extremely relevant. More than one out of three cases treated (35 (41.2\%) out of 85 ) experienced side-effects, with 27 (31.8\%) having adverse reactions serious enough to indicate drug discontinuation.

Our data showed a significant difference in side-effects between a $600 \mathrm{mg}$ and 1,200 $\mathrm{mg}$ daily dosage of linezolid: they were less frequent when the lower dosage was prescribed, whereas no significant difference was seen in efficacy between the two doses. These data suggest that a lower dose is sufficient to convey the possible benefits of linezolid while

\begin{tabular}{|c|c|c|c|c|}
\hline \multirow[t]{2}{*}{ TABLE 2} & \multicolumn{4}{|c|}{$\begin{array}{l}\text { Comparison of efficacy end-points for the } \\
\text { treatment of multidrug-resistant/extensively drug- } \\
\text { resistant tuberculosis with or without linezolid in } \\
\text { cases with known outcome }\end{array}$} \\
\hline & & Linezolid & No linezolid & $p$-value \\
\hline Patients $\mathbf{n}$ & & 45 & 110 & \\
\hline \multicolumn{5}{|c|}{$\begin{array}{l}\text { Sputum smear conversion } \\
\text { time days }\end{array}$} \\
\hline Mean $\pm S D$ & & $102.9 \pm 74$ & $65.4 \pm 80.1$ & 0.007 \\
\hline n (\%) & & $31(69)$ & $59(54)$ & \\
\hline \multicolumn{5}{|c|}{$\begin{array}{l}\text { Culture conversion time } \\
\text { days }\end{array}$} \\
\hline Mean \pm SD & & $109 \pm 71$ & $69 \pm 63$ & 0.0007 \\
\hline n (\%) & & 39 (87) & $86(78)$ & \\
\hline \multicolumn{5}{|c|}{ Treatment outcome } \\
\hline Success & & $36(80.0)$ & $90(81.8)$ & 0.88 \\
\hline Cured & & $23(51.1)$ & $75(68.2)$ & 0.04 \\
\hline Completed & & $13(28.9)$ & 15 (13.6) & 0.02 \\
\hline Failure & & & $1(0.9)$ & \\
\hline Death & & $9(20)$ & 19 (17.3) & 0.65 \\
\hline
\end{tabular}

reducing the risk of major side-effects. In contrast, based on a case series of eight patients treated with $600 \mathrm{mg}$ daily of linezolid, PARK et al. [33] suggested that this "daily half dose" was effective in treating intractable MDR/XDR-TB cases, but did not prevent appearance of major adverse events.

In our cohort, linezolid was administered for a mean duration exceeding 32 weeks. Thirteen (37.1\%) episodes of linezolidattributed adverse effects occurred in the first 8 weeks of treatment. Only one $(3.6 \%)$ out of 28 patients who received $600 \mathrm{mg}$ of linezolid daily experienced adverse drug effects in the first 8 weeks of treatment. Adverse effects appeared after a median time of $\sim 10$ weeks ( 69 days), suggesting that after $\sim 2$ months of linezolid treatment, the risk of adverse events increases.

Our data show that patients treated with linezolid (at either dose) achieved similar treatment outcomes to those in the nonlinezolid group, although those treated with linezolid had a more frequent history of previous treatment and were resistant to more first- and second-line drugs than patients who were not. Patients treated with linezolid achieved a higher proportion of sputum smear and culture conversion (significant for resistance to more than seven drugs only), but took significantly longer to convert than patients who were not treated with linezolid. This apparent increase in treatment success in XDR-TB patients might represent an opportunity for decreasing transmission of XDR-TB strains. It would be useful to improve the methodology in future studies (e.g. prospective, randomised or blinded evaluation) in order to assess the safety, efficacy and quality of the drug more thoroughly. These findings suggest that linezolid may confer a benefit for treatment of the most complicated cases of MDR/XDR-TB, and warrants further study in a randomised, controlled trial.

To our knowledge, this is the first study allowing a comprehensive evaluation of linezolid tolerability and a first 
TABLE 3 Proportion of converters, according to the number of antituberculous drugs to which isolates were resistant, among patients treated with and without linezolid (LZD) in Belarus, Germany, Italy and Switzerland, 2001-2007

\begin{tabular}{|c|c|c|c|c|c|c|}
\hline Drug resistance $n$ & \multicolumn{3}{|c|}{ Sputum smear conversion } & \multicolumn{3}{|c|}{ Culture conversion } \\
\hline$<5$ & $6 / 9(66.7)$ & 35/59 (59.3) & 0.68 & 8/9 (88.9) & $50 / 59(84.7)$ & 0.75 \\
\hline $5-7$ & $16 / 26(61.5)$ & 23/43 (53.5) & 0.51 & 22/26 (84.6) & $34 / 43(79.1)$ & 0.6 \\
\hline$>7$ & $9 / 10(90)$ & $1 / 8(12.5)$ & 0.0009 & $9 / 10(90)$ & 2/8 (25) & 0.004 \\
\hline Total & $31 / 45(68.9)$ & $59 / 110(53.6)$ & 0.07 & $39 / 45(86.7)$ & $86 / 110(78.2)$ & 0.22 \\
\hline
\end{tabular}

Data are presented as $n / n(\%)$, unless otherwise stated.

report on its long-term efficacy in a clinical setting. The study strengths include a relatively large sample size, the quality of laboratory data (all XDR-TB-defining drugs tested and qualitycontrolled DST) and the capacity to provide representative data from four European countries.

The study has several limitations associated with its retrospective, observational nature. Although the described adverse effects were reversible in all cases when therapy with linezolid was discontinued, cause and effect of linezolid therapy and adverse effects are not proven, as they may also be due to other drugs. Another bias is the nonrandomised allocation of patients to linezolid treatment. As a consequence of the linezolid-treated patients being resistant to more drugs than those not treated with linezolid (and therefore presumably having a poorer chance of treatment success), the effect of linezolid on treatment outcome may have been diminished in these data. In addition, even with a relatively sizable cohort of patients, numbers of patients when data were stratified by resistance pattern became small, challenging our ability to evaluate the potential effects of linezolid on efficacy in a definitive manner.

The study results are important in that they 1) confirm the substantial risk of adverse effects noted in other studies of linezolid, but suggest a safer dosage that could be prescribed without losing the potential benefits of linezolid; 2) provide documentation for cautiously justifying the use of linezolid in clinical practice for only the most complicated MDR/XDR-TB cases; 3) contribute evidence for regulatory authorities to develop clear indications for the use of linezolid in MDR/ XDR-TB treatment; 4) represent a baseline against which future studies can be compared; and 5) allow for justification and improved planning of prospective, controlled clinical trials. Overall, the study results have both clinical and public health relevance.

In conclusion, the data suggest that linezolid may be useful in improving the chances of smear and culture conversion and may provide a better chance at treatment success in only the most complicated cases of MDR/XDR-TB when other treatment alternatives are not available, but that its safety profile does not warrant its use in cases for which other, safer secondline or third-line drugs are available. The side-effects associated with linezolid are frequent and often serious, and require careful monitoring, particularly when treatment extends beyond 8 weeks. It is likely that the potential benefits of linezolid can be achieved using a lower dosage of $600 \mathrm{mg}$ q.d., which appeared to reduce the risk of side-effects in this cohort of patients, therefore reducing costs without changing efficacy. Additional randomised, controlled trials of linezolid for difficult-to-treat cases of MDR/XDR-TB should be conducted to confirm these findings.

\section{SUPPORT STATEMENT}

This study was supported by the current research funds of the participating institutions. The data collection system was initiated in 1996 with funding obtained by the Italian Association of Hospital Pulmonologists (AIPO) through a Ministry of Health/Superior Institute of Health grant (National TB Project, Grant No. 1, 641/96). The study is partially funded by the European Respiratory Society as a Clinical Research Collaboration.

\section{STATEMENT OF INTEREST}

None declared.

TABLE 4 Logistic regression analysis of potential independent variables associated with adverse treatment outcome (failure or death) in multidrug-resistant/extensively drug-resistant tuberculosis (XDR-TB) cases

\begin{tabular}{|c|c|c|c|c|}
\hline Variables & Crude odds ratio & p-value & Adjusted odds ratio & p-value \\
\hline Sex male & $1.11(0.43-2.85)$ & 0.82 & $1.8(0.56-5.78)$ & 0.32 \\
\hline Increasing age yrs & $1.03(1.008-1.06)$ & 0.01 & $1.03(1.003-1.07)$ & 0.03 \\
\hline Previous treatment $>\mathbf{3 0}$ days & $1.8(0.76-4.27)$ & 0.18 & $1.01(0.38-2.65)$ & 0.98 \\
\hline Resistance to $>5$ drugs & $2.67(1.17-6.1)$ & 0.01 & $1.78(0.59-5.34)$ & 0.29 \\
\hline Linezolid treatment & $0.88(0.36-2.13)$ & 0.79 & $1.1(0.39-3.1)$ & 0.85 \\
\hline XDR-TB & $11.6(3.2-42.05)$ & $<0.0001$ & $8.3(1.74-40.28)$ & 0.008 \\
\hline
\end{tabular}

Data are presented as odds ratio (95\% confidence interval), unless otherwise stated. 


\section{ACKNOWLEDGEMENTS}

The authors' affiliations are as follows. G.B. Migliori and R. Centis: WHO Collaborating Centre for TB and Lung Diseases, Fondazione S. Maugeri, Care and Research Institute, Tradate, Italy. B. Eker and C. Lange: Division of Clinical Infectious Diseases, Research Center Borstel, Borstel, Germany. M.D. Richardson: HIV/TB Global Program, PATH, Seattle, WA, USA. G. Sotgiu: Hygiene and Preventive Medicine Institute, University of Sassari, Sassari, Italy. J-P. Zellweger: TB Clinic, Dept of Ambulatory Care and Community Medicine, University of Lausanne, Lausanne, Switzerland. A. Skrahina: Research Institute of Pulmonology and Tuberculosis, Minsk, Belarus. J. Ortmann: Pulmonary Clinic, Karl-Hansen-Clinic, Bad Lippspringe, Germany. E. Girardi and N. Bevilacqua: National Institute for Infectious Diseases (INMI) L. Spallanzani, Rome, Italy. H. Hoffmann: Supranational Reference Laboratory of Tuberculosis, Asklepios Clinic MunichGauting, Munich-Gauting, Germany. G. Besozzi: E. Morelli Hospital, Reference Hospital for MDR and HIV TB, Sondalo, Italy. D. Kirsten: Hospital Großhansdorf, Center for Pneumology and Thoracic Surgery, Großhansdorf, Germany.

The additional participating members of the TBNET Study Group are as follows. Belarus: H. Hurevich, Research Institute of Pulmonology and Tuberculosis, Minsk; A. Skrahin, Belarus State Medical University, Minsk. Germany: R. Muetterlein, Regional Hospital, Pasberg; T. Schaberg, Pulmonary Clinic, Deakoness Hospital, Rotenburg; R. Eberhardt, Division of Pulmonary Medicine, Thorax-ClinicHeidelberg, Heidelberg; H. Flick, Division of Infectious Diseases, Charité Berlin, Berlin; A. de Roux, Pulmonary Clinic, Protestant Pulmonary Clinic, Berlin; M. Hamm, Pulmonary Clinic, Hospital Diekholzen, Diekholzen; H. Hang, Pulmonary Clinic, Hospital Schillerhöhe, Schillerhöhe; P. Hannemann, Pulmonary Clinic, Deakoness Hospital, Neustadt; D. Hillemann, Division of Mycobacteriology, Research Center Borstel, Borstel; B. Kretz, Asklepios Pulmonary Hospital, Gauting; C. Laumanns, Bethania Hospital, Solingen; A. Paulick, Pulmonary Clinic-Coswig, Coswig; M.W. Pletz, Division of Pulmonary Medicine, Hanover Medical School, Hanover; M. Rau, Pulmonary Clinic, Wangen; C. Schaudt, Pulmonary Clinic, Hospital St Blasien, St Blasien. Italy: A. Matteelli, Brescia University, Brescia; A. Spanevello, Insubria University, Varese; O. Toungoussova, Fondazione S. Maugeri, Cassano delle Murge; Lia D'Ambrosio, Fondazione S. Maugeri, Tradate; S. De Lorenzo, P. Troupioti and G. De Iaco, Sondalo Hospital, Sondalo; A. Gualano, P. De Mori and F.N. Lauria, INMI L. Spallanzani, Rome; G. Ferrara, University of Perugia, Perugia; D. Cirillo, S. Raffaele Hospital, Milan. Switzerland: J.P. Janssens, University Hospital Geneva, Geneva.

\section{REFERENCES}

1 World Health Organization, Global Tuberculosis Control Surveillance, Planning, Financing. WHO Report 2008. WHO/ HTM/TB/2008.393. Geneva, World Health Organization, 2008.

2 Zignol M, Hosseini MS, Wright A, et al. Global incidence of multidrug-resistant tuberculosis. J Infect Dis 2006; 194: 479-485.

3 Aziz MA, Wright A, Laszlo A, et al. Epidemiology of antituberculosis drug resistance (the Global Project on Anti-tuberculosis Drug Resistance Surveillance): an updated analysis. Lancet 2006; 368: 2142-2154.

4 Espinal MA, Laszlo A, Simonsen L, et al. Global trends in resistance to antituberculosis drugs. World Health OrganizationInternational Union against Tuberculosis and Lung Disease Working Group on Anti-Tuberculosis Drug Resistance Surveillance. N Engl J Med 2001; 344: 1294-1303.

5 Raviglione MC, Uplekar MW. WHO's new Stop TB Strategy. Lancet 2006; 367: 952-955.

6 World Health Organization, The WHO/IUATLD Global Project on Anti-Tuberculosis Drug Resistance Surveillance. Anti-tuberculosis
Drug Resistance in the World: Fourth Global Report. WHO/HTM/ TB/2008.394. Geneva, World Health Organization, 2008.

7 Extensively drug-resistant tuberculosis (XDR-TB), recommendations for prevention and control. Wkly Epidemiol Rec 2006; 81: 430-432.

8 Centers for Disease Control and Prevention, Emergence of Mycobacterium tuberculosis with extensive resistance to second-line drugs - worldwide, 2000-2004. MMWR Morb Mortal Wkly Rep 2006; 55: 301-305.

9 Migliori GB, Ortmann J, Girardi E, et al. Extensively drug-resistant tuberculosis, Italy and Germany. Emerg Infect Dis 2007; 13: 780-782.

10 Migliori GB, Besozzi G, Girardi E, et al. Clinical and operational value of the extensively drug-resistant tuberculosis definition. Eur Respir J 2007; 30: 623-626.

11 Migliori GB, Lange C, Girardi E, et al. Extensively drug-resistant tuberculosis is worse than multidrug-resistant tuberculosis: different methodology and settings, same results. Clin Infect Dis 2008; 46: 958-959.

12 Kim HR, Hwang SS, Kim HJ, et al. Impact of extensive drug resistance on treatment outcomes in non-HIV-infected patients with multidrug-resistant tuberculosis. Clin Infect Dis 2007; 45: 1290-1295.

13 Migliori GB, Lange C, Girardi E, et al. Fluoroquinolones: are they essential to treat multidrug-resistant tuberculosis? Eur Respir 2008; 31: 904-905.

14 Migliori GB, Lange C, Centis R, et al. Resistance to second-line injectables and treatment outcomes in multidrug-resistant and extensively drug-resistant tuberculosis cases. Eur Respir J 2008; 31: $1155-1159$.

15 Eker B, Ortmann J, Migliori GB, et al. Multidrug- and extensively drug-resistant tuberculosis, Germany. Emerg Infect Dis 2008; 14: 1700-1706.

16 Molicotti P, Ortu S, Bua A, et al. In vitro efficacy of linezolid on clinical strains of Mycobacterium tuberculosis and other mycobacteria. New Microbiol 2006; 29: 275-280.

17 Tato M, de la Pedrosa EG, Canton R, et al. In vitro activity of linezolid against Mycobacterium tuberculosis complex, including multidrug-resistant Mycobacterium bovis isolates. Int J Antimicrob Agents 2006; 28: 75-78.

18 Erturan Z, Uzun M. In vitro activity of linezolid against multidrugresistant Mycobacterium tuberculosis isolates. Int J Antimicrob Agents 2005; 26: 78-80.

19 Alcala L, Ruiz-Serrano MJ, Perez-Fernandez Turegano C, et al. In vitro activities of linezolid against clinical isolates of Mycobacterium tuberculosis that are susceptible or resistant to first-line antituberculous drugs. Antimicrob Agents Chemother 2003; 47: 416-417.

20 Rodriguez JC, Ruiz M, Lopez $\mathrm{M}$, et al. In vitro activity of moxifloxacin, levofloxacin, gatifloxacin and linezolid against Mycobacterium tuberculosis. Int J Antimicrob Agents 2002; 20: 464-467.

21 Diekema DJ, Jones RN. Oxazolidinone antibiotics. Lancet 2001; 358: 1975-1982.

22 Fortun J, Martin-Davila P, Navas E, et al. Linezolid for the treatment of multidrug-resistant tuberculosis. J Antimicrob Chemother 2005; 56: 180-185.

23 Dietze R, Hadad DJ, McGee B, et al. Early and extended early bactericidal activity of linezolid in pulmonary tuberculosis. Am J Respir Crit Care Med 2008; 178: 1180-1185.

24 Nannini EC, Keating M, Binstock P, et al. Successful treatment of refractory disseminated Mycobacterium avium complex infection with the addition of linezolid and mefloquine. J Infect 2002; 44: 201-203.

25 Brown-Elliott BA, Wallace RJ Jr, Blinkhorn R, et al. Successful treatment of disseminated Mycobacterium chelonae infection with linezolid. Clin Infect Dis 2001; 33: 1433-1434. 
26 Morales P, Ros JA, Blanes M, et al. Successful recovery after disseminated infection due to mycobacterium abscesses in a lung transplant patient: subcutaneous nodule as first manifestation - a case report. Transplant Proc 2007; 39: 2413-2415.

27 Hoetzenecker W, Ulmer A, Klingel K, et al. Dissemination of a localized cutaneous infection with Mycobacterium chelonae under immunosuppressive treatment. Arch Dermatol 2007; 143: 951-952.

28 Kyle SD, Porter WM. Mycobacterium chelonae infection successfully treated with oral clarithromycin and linezolid. Br J Dermatol 2004; 151: 1101.

29 Ntziora F, Falagas ME. Linezolid for the treatment of patients with atypical mycobacterial infection: a systematic review. Int J Tuberc Lung Dis 2007; 11: 606-611.

30 Condos R, Hadgiangelis N, Leibert E, et al. Case series report of a linezolid-containing regimen for extensively drug-resistant tuberculosis. Chest 2008; 134: 187-192.
31 Yew WW, Chau $\mathrm{CH}$, Wen KH. Linezolid in the treatment of "difficult" multidrug-resistant tuberculosis. Int J Tuberc Lung Dis 2008; 12: 345-346.

32 von der Lippe B, Sandven P, Brubakk O. Efficacy and safety of linezolid in multidrug resistant tuberculosis (MDR-TB) - a report of ten cases. J Infect 2006; 52: 92-96.

33 Park IN, Hong SB, Oh YM, et al. Efficacy and tolerability of daily-half dose linezolid in patients with intractable multidrug-resistant tuberculosis. J Antimicrob Chemother 2006; 58: 701-704.

34 Laserson KF, Thorpe LE, Leimane V, et al. Speaking the same language: treatment outcome definitions for multidrug-resistant tuberculosis. Int J Tuberc Lung Dis 2005; 9: 640-645.

35 Sotgiu G, Ferrara G, Matteelli A, et al. Epidemiology and clinical management of XDR-TB: a systematic review by TBNET. Eur Respir J 2009; 33: 871-881. 